\title{
Detection of significant pathways in osteoporosis based on graph clustering
}

\author{
HAIJUN XIAO, LIANCHENG SHAN, HAIMING ZHU and FENG XUE \\ Department of Orthopedics, Fengxian Central Hospital, Shanghai 201400, P.R. China
}

Received April 9, 2012; Accepted August 8, 2012

DOI: $10.3892 / \mathrm{mmr} .2012 .1082$

\begin{abstract}
Osteoporosis is the most common and serious skeletal disorder among the elderly, characterized by a low bone mineral density (BMD). Low bone mass in the elderly is highly dependent on their peak bone mass (PBM) as young adults. Circulating monocytes serve as early progenitors of osteoclasts and produce significant molecules for bone metabolism. An improved understanding of the biology and genetics of osteoclast differentiation at the pathway level is likely to be beneficial for the development of novel targeted approaches for osteoporosis. The objective of this study was to explore gene expression profiles comprehensively by grouping individual differentially expressed genes (DEGs) into gene sets and pathways using the graph clustering approach and Gene Ontology (GO) term enrichment analysis. The results indicated that the DEGs between high and low PBM samples were grouped into nine gene sets. The genes in clusters 1 and 8 (including GBP1, STAT1, CXCL10 and EIF2AK2) may be associated with osteoclast differentiation by the immune system response. The genes in clusters 2, 7 and 9 (including SOCS3, SOD2, ATF3, ADM EGR2 and BCL2A1) may be associated with osteoclast differentiation by responses to various stimuli. This study provides a number of candidate genes that warrant further investigation, including DDX60, HERC5, RSAD2, SIGLEC1, CMPK2, MX1, SEPING1, EPSTI1, C9orf72, PHLDA2, PFKFB3, PLEKHG2, ANKRD28, IL1RN and RNF19B.
\end{abstract}

\section{Introduction}

Osteoporosis is the most common and serious skeletal disorder among the elderly. Symptomatic osteoporosis occurs due to a decreased bone mineral density (BMD) leading to reduced bone strength and an increased risk of fractures (1). Low bone mass in the elderly is highly dependent on their peak bone mass (PBM) as young adults (2). Therefore, it is necessary to

Correspondence to: Mr Xue Feng, Department of Orthopedics, Fengxian Central Hospital, 9588 Nanfeng Road, Fengxian, Shanghai 201400, P.R. China.

E-mail: xuefengssdd@hotmail.com

Key words: differentially expressed genes, graph cluster, osteoporosis, peak bone mass, significant pathways understand and identify the risk factors for impaired PBM in young and middle-aged adults.

Osteopenia may result from an imbalance between increased bone resorption and decreased bone formation $(3,4)$. Bone resorption involves the dissolution of bone mineral and degradation of the organic bone matrix. These two functions are performed by osteoclasts. Osteoclasts are members of the monocyte/macrophage lineage and are formed by multiple instances of cellular fusion of their mononuclear precursors (5). Monocytes differentiate into osteoclasts in the presence of various molecular signals (6). RANKL, one of the most frequently studied, is a ligand for the receptor activator of nuclear factor- $\kappa \mathrm{B}(\mathrm{NF}-\kappa \mathrm{B}$; RANK) on osteoclast precursor cells (7). RANKL/RANK signaling activates four pathways that mediate osteoclast formation; NF- $\kappa \mathrm{B}$, c-fos and calcineurin/NFATc1 and three pathways that mediate osteoclast activation; Src and MKK6/p38/MITF and survival; Src and extracellular signal-regulated kinase (8). Osteoblasts produce and secrete osteoprotegerin, a decoy receptor that binds to RANKL and blocks RANKL/RANK interactions and hence suppresses the ability of RANK to increase bone resorption (9). Previous studies have shown that blood monocytes also produce a wide variety of inflammatory factors and transcription factors involved in bone metabolism, including interleukin-1 (10), tumor necrosis factor- $\alpha$ (TNF- $\alpha$ ) (11), interleukin-6 (12), platelet-derived growth factor (13), transforming growth factor- $\beta$ (14), resolvinE1 (15), runt-related transcription factor 2 (Runx $2 ; 16$ ), guanylate binding protein 1 (GBP1), signal transducer and activator of transcription 1 (STAT1), CXC chemokine ligand 10 (CXCL10) (17), chemokine receptor 3 , histidine decarboxylase and glucocorticoid receptor genes (18).

However, it is unknown whether other mechanisms regulating these factors are significant in the ability of monocytes to affect bone metabolism. Since biological processes are mediated by multiple, co-regulated genes working in synchrony, certain unknown genes may be assigned potential biological functions when studied in gene sets with known genes and ontology groups (19). Thus, the objective of this study was to screen the differential gene expression in monocytes using a highthroughput microarray platform and to explore gene expression profiles comprehensively by grouping individual differentially expressed genes (DEGs) into gene sets and Gene Ontology (GO) terms. The DEGs between high and low PBM samples were grouped into nine gene sets using the graph-clustering 
approach. GO term enrichment analysis was applied to identify the relevant molecular functions in response to an impaired PBM. The current study revealed that the DEGs, as precursors of osteoclasts, are functionally involved in the immune response. The stimulus response may contribute to differential osteoclastogenesis, leading to differential PBM levels.

\section{Materials and methods}

Affymetrix microarray data. Circulating monocyte affymetrix microarray datasets were accessible from the National Center for Biotechnology Information Gene Expression Omnibus (GEO) data repository (http://www.ncbi.nlm.nih.gov/geo/) using the series accession number GSE7158. Fourteen subjects with extremely high PBM levels and 12 subjects with extremely low PBM levels were selected for DNA microarray experiments. All the recruited volunteers signed an informed consent form prior to entering this project.

Statistical analysis. The limma method (20) was used to identify DEGs. The raw expression datasets from all conditions were normalized using the Robust Multiarray Average (RMA) method with the default settings implemented in Bioconductor and then the linear model was constructed. DEGs with a fold change $>1.5$ and $\mathrm{P}<0.05$ were selected.

The Pearson correlation coefficient (r) was used to compare the potential correlations between DEGs. Statistical significance was set at $r>0.95$ and $P<0.05$. All statistical tests were performed using R language (21).

Network analyses and graph clustering. To identify co-expressed groups, DPClus, a graph clustering algorithm that extracts densely connected nodes as a cluster, was used (22). DPClus is based on the density and periphery tracking of clusters and is freely available from http://kanaya. naist.jp/DPClus/. In the current study, the overlapping mode with the DPClus settings were used. The parameter settings of cluster properties were set; density values were set to 0.5 (23) and minimum cluster size was set to 2 .

GO term enrichment analysis. The GO (24) project is a major bioinformatics initiative with the aim of standardizing the representation of genes and gene product attributes across species and databases. The project provides a controlled vocabulary of terms for describing gene product characteristics and gene product annotation data from GO Consortium members, as well as tools to access and process this data.

The DAVID tool (25) was used to identify overrepresented GO terms in biological process. $\mathrm{P}<0.05$ and counts of $>2$ were set as the threshold for the analysis using the hypergeometric distribution.

\section{Results}

Differential gene expression profiling and co-expression network construction. GSE7158 microarray datasets were publicly available from the GEO database. Following microarray analysis, a total of 49 genes were selected as DEGs with a fold change $>1.5$ and $\mathrm{P}<0.05$. The expression profiling of these 49 DEGs is presented in Fig. 1.
To form the correlations between DEGs, $r>0.7$ and $\mathrm{P}<0.05$ were selected as the cut-off points. A correlation network was constructed with a total of 159 correlations among 49 DEGs (Fig. 2).

Graph clustering identifies modules significantly enriched for DEGs contained in GO term pathways. At r $>0.7$, DPClus (22) identified 9 clusters in the correlation network for osteoporosis, ranging in size from 3-14 genes. Clusters 1, 2, 7, 8 and 9 were connected as they shared the same genes. For example, one gene (epithelial stromal interaction 1, EPSTI1) was shared between clusters 1 and 8; three genes (suppressor of cytokine signaling, SOCS3; superoxide dismutase, SOD2 and activating transcription factor 3 , ATF3) were shared between cluster 2 and 7 and one gene (adrenomedullin, ADM) was shared between clusters 2 and 9. The higher the number of genes shared, the more connectivity among them (corresponding to the thicker lines; Fig. 3).

To assess the significance of the obtained clusters, the overrepresented GO terms were used. Enrichment analysis was performed using the hypergeometrical distribution to find the significant GO term enrichment pathways. In accordance with the graph clustering results, the genes in clusters 1 and 8 were enriched in similar pathways, including immune responses and circulatory system processes. The genes in clusters 2, 7 and 9 were enriched in similar pathways regulating apoptosis and responding to various stimuli, including insulin, hypoxia, nutrients, drugs, radiation and hormones (Table I). Clusters 2 and 7 had the most similar GO term enrichment pathways. These GO biological processes may be relevant to the differentiation of monocytes into osteoclasts.

\section{Discussion}

In the current study, differential expression profiling was systematically investigated and its possible role in the differentiation of osteoclasts was explored. A total of 49 DEGs were identified and correlated to produce 159 network connections. These DEGs were assigned into nine clusters using the graph clustering method in response to different PBM levels. A total of 14 genes were included in cluster 1 [GBP1; interferoninduced protein with tetratricopeptide repeats 2, IFIT2; eukaryotic translation initiation factor $2-\alpha$ kinase 2, EIF2AK2; interferon-induced protein 44, IFI44; IFI44L; DEAD (Asp-Glu-Ala-Asp) box polypeptide 60, DDX60; HECT and RLD domain containing E3 ubiquitin protein ligase 5, HERC5; radical S-adenosyl methionine domain containing 2, RSAD2; sialic acid binding Ig-like lectin 1, sialoadhesin, SIGLEC1; cytidine monophosphate kinase 2, CMPK2; EPSTI1; interferon, $\alpha$-inducible protein 6, IFI6; CXCL10; and myxovirus resistance 1 , interferon-inducible protein $\mathrm{p} 78, \mathrm{MX} 1]$ and 3 genes were involved in cluster 8 (STAT1; EPSTI1; and serpin peptidase inhibitor, clade G, SERPING1). Notably, cluster 8 was connected with all the genes of cluster 1 by STAT1 and EPSTI1 in order to be involved in immune responses and circulatory system processes, as demonstrated in previous studies.

The immune system has been correlated with bone resorption through a complex interaction involving $\mathrm{T}$ and B lymphocytes, dendritic cells (DCs), cytokines and cell-cell interactions (26). There is strong evidence that STAT1 is 


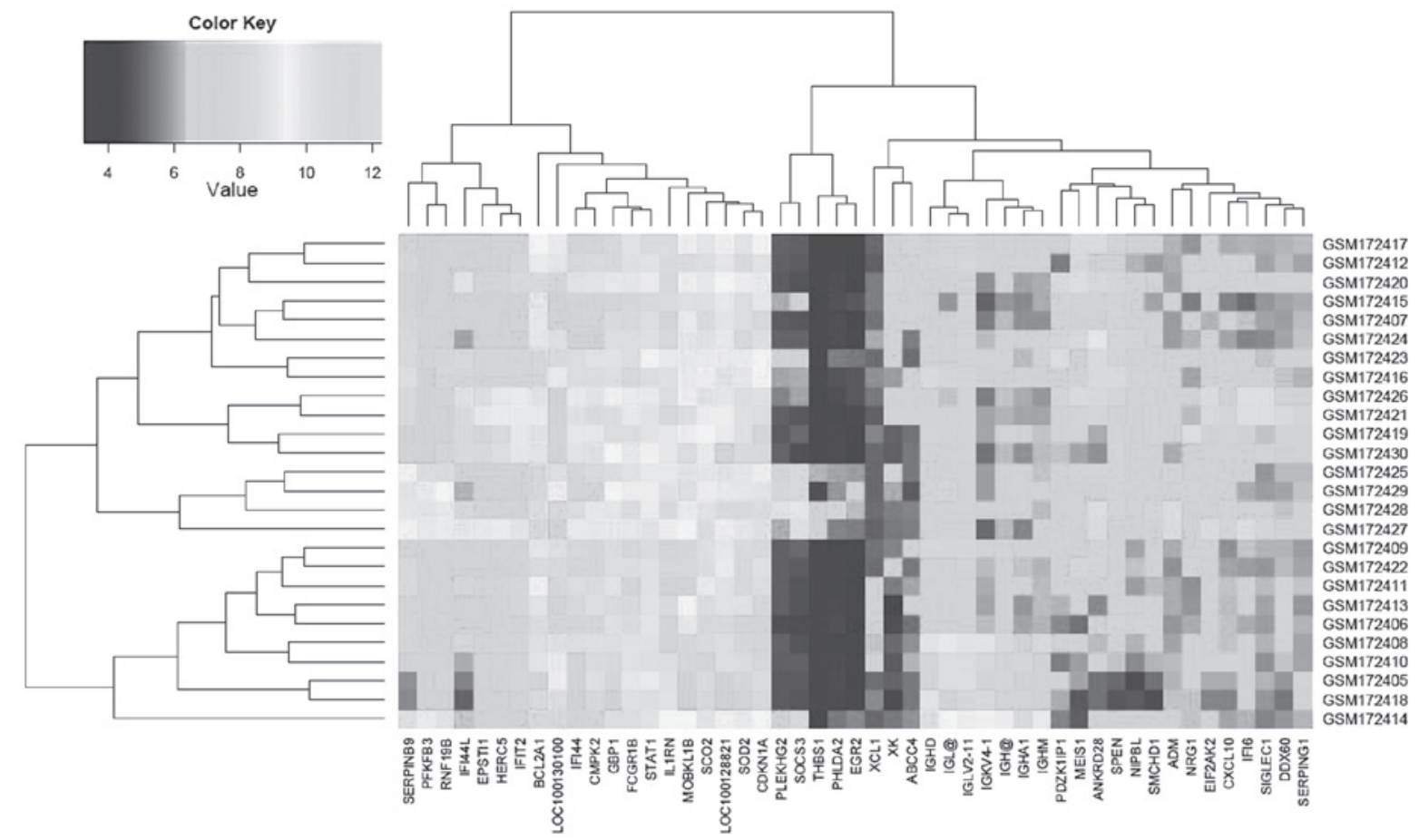

Figure 1. The expression profiling of 49 DEGs. Each row represents the samples and each line represents the expression values of the DEGs. Black indicates low expression, gray indicates medium expression and white indicates high expression. DEGs, differentially expressed genes.

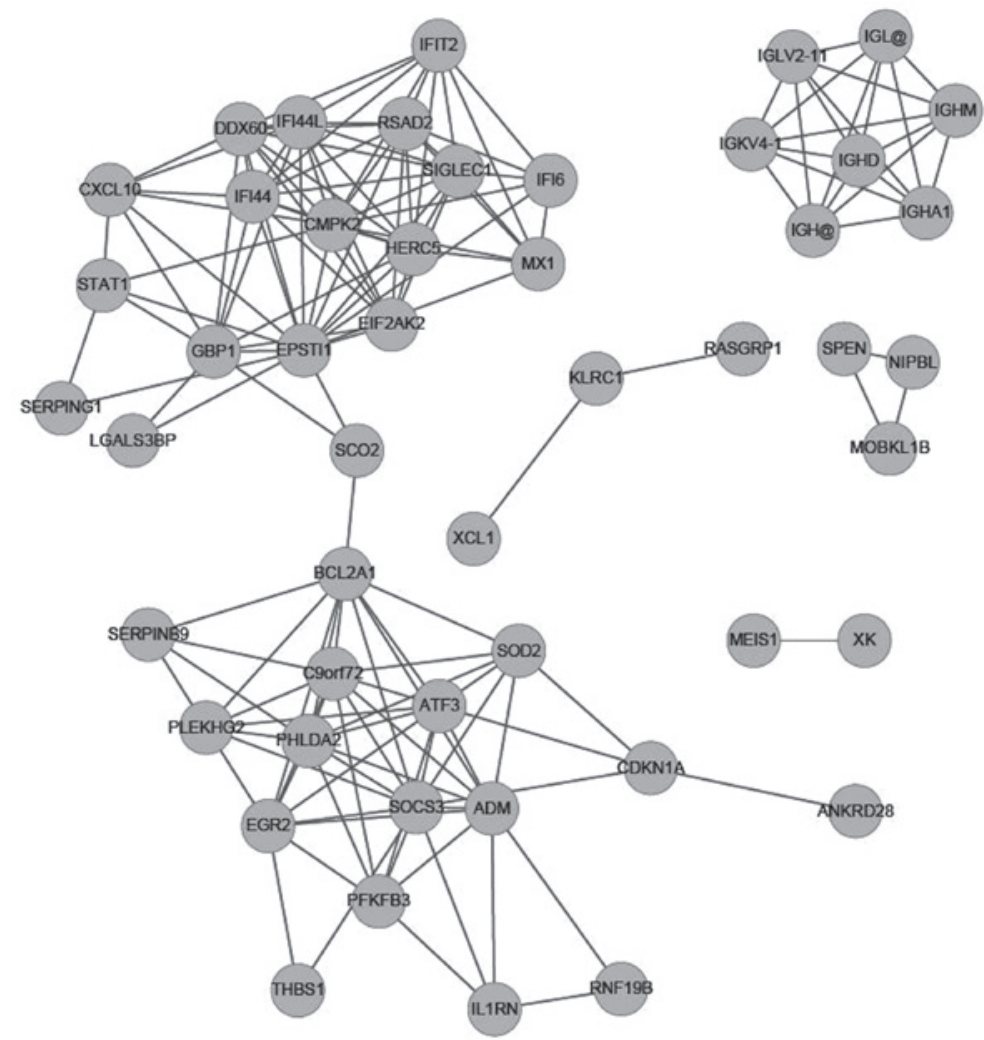

Figure 2. Co-expression network of osteoporosis. A total of 159 correlations with $r>0.7$ and $\mathrm{P}<0.05$ are exhibited. The nodes indicate the DEGs and the links indicate the high correlation among the DEGs. DEGs, differentially expressed genes.

significant in bone metabolism as STAT1 has been reported to be upregulated in the femur tissue of osteoporotic mice (27) and humans (18). STAT1 may serve as a primary mediator of interferon (IFN) signaling pathways involving osteoclast differentiation. Through the p38 MAPK pathway, RANKL stimulates the serine phosphorylation of STAT1, resulting in the migration and adhesion of osteoclast precursors (28). STAT1 interacts with Runx2, an essential transcription factor 
Table I. List of enriched GO terms in clusters 1, 2, 7, 8 and 9 detected by DPClus.

\begin{tabular}{|c|c|c|c|c|c|}
\hline Category & Term & Description & Count & P-value & FDR \\
\hline \multirow[t]{3}{*}{ Cluster 1} & GO:0009615 & Response to virus & 4 & $5.86 \mathrm{e}^{-5}$ & 0.006833 \\
\hline & GO:0006955 & Immune response & 5 & 0.00110057 & 0.062388 \\
\hline & GO:0006952 & Defense response & 4 & 0.00882795 & 0.292359 \\
\hline \multirow[t]{35}{*}{ Cluster 2} & GO:0032868 & Response to insulin stimulus & 3 & 0.00147149 & 0.378515 \\
\hline & GO:0007568 & Aging & 3 & 0.00177687 & 0.249653 \\
\hline & GO:0001666 & Response to hypoxia & 3 & 0.00262243 & 0.246268 \\
\hline & GO:0070482 & Response to oxygen levels & 3 & 0.00289863 & 0.208958 \\
\hline & GO:0043434 & Response to peptide hormone stimulus & 3 & 0.00344654 & 0.19991 \\
\hline & GO:0006915 & Apoptosis & 4 & 0.00415134 & 0.200641 \\
\hline & GO:0012501 & Programmed cell death & 4 & 0.00432956 & 0.181443 \\
\hline & GO:0031667 & Response to nutrient levels & 3 & 0.00557639 & 0.202104 \\
\hline & GO:0006916 & Anti-apoptosis & 3 & 0.00608264 & 0.196651 \\
\hline & GO:0008219 & Cell death & 4 & 0.00684652 & 0.199007 \\
\hline & GO:0009991 & Response to extracellular stimulus & 3 & 0.00691087 & 0.184238 \\
\hline & GO:0016265 & Death & 4 & 0.00698058 & 0.171843 \\
\hline & GO:0042981 & Regulation of apoptosis & 4 & 0.00934738 & 0.208115 \\
\hline & GO:0043067 & Regulation of programmed cell death & 4 & 0.00960751 & 0.19967 \\
\hline & GO:0010941 & Regulation of cell death & 4 & 0.00970618 & 0.189438 \\
\hline & GO:0010332 & Response to gamma radiation & 2 & 0.01352424 & 0.240339 \\
\hline & GO:0031100 & Organ regeneration & 2 & 0.01527641 & 0.253599 \\
\hline & GO:0048666 & Neuron development & 3 & 0.01586432 & 0.249457 \\
\hline & GO:0043066 & Negative regulation of apoptosis & 3 & 0.01722442 & 0.255741 \\
\hline & GO:0043069 & Negative regulation of programmed cell death & 3 & 0.01768878 & 0.250411 \\
\hline & GO:0060548 & Negative regulation of cell death & 3 & 0.01778231 & 0.241164 \\
\hline & GO:0009725 & Response to hormone stimulus & 3 & 0.01844308 & 0.23914 \\
\hline & GO:0009628 & Response to abiotic stimulus & 3 & 0.01853835 & 0.231094 \\
\hline & GO:0006873 & Cellular ion homeostasis & 3 & 0.01911445 & 0.228747 \\
\hline & GO:0055082 & Cellular chemical homeostasis & 3 & 0.01969829 & 0.226664 \\
\hline & GO:0009719 & Response to endogenous stimulus & 3 & 0.0222131 & 0.24351 \\
\hline & GO:0050801 & Ion homeostasis & 3 & 0.02262762 & 0.239518 \\
\hline & GO:0030182 & Neuron differentiation & 3 & 0.02573112 & 0.259709 \\
\hline & GO:0019725 & Cellular homeostasis & 3 & 0.02888801 & 0.27855 \\
\hline & GO:0048878 & Chemical homeostasis & 3 & 0.03440423 & 0.314043 \\
\hline & GO:0010212 & Response to ionizing radiation & 2 & 0.03494492 & 0.309692 \\
\hline & GO:0031099 & Regeneration & 2 & 0.0400934 & 0.338357 \\
\hline & GO:0032496 & Response to lipopolysaccharide & 2 & 0.04464963 & 0.360509 \\
\hline & GO:0009266 & Response to temperature stimulus & 2 & 0.04805437 & 0.373652 \\
\hline & GO:0002237 & Response to molecule of bacterial origin & 2 & 0.04975276 & 0.375599 \\
\hline \multirow[t]{14}{*}{ Cluster 7} & GO:0070482 & Response to oxygen levels & 3 & $3.21 \mathrm{e}^{-4}$ & 0.079885 \\
\hline & GO:0009314 & Response to radiation & 3 & $6.46 \mathrm{e}^{-4}$ & 0.080292 \\
\hline & GO:0042493 & Response to drug & 3 & $7.53 \mathrm{e}^{-4}$ & 0.062989 \\
\hline & GO:0009991 & Response to extracellular stimulus & 3 & $7.81 \mathrm{e}^{-4}$ & 0.049354 \\
\hline & GO:0055093 & Response to hyperoxia & 2 & 0.00177318 & 0.087833 \\
\hline & GO:0043066 & Negative regulation of apoptosis & 3 & 0.00201309 & 0.08331 \\
\hline & GO:0043069 & Negative regulation of programmed cell death & 3 & 0.00206992 & 0.073801 \\
\hline & GO:0060548 & Negative regulation of cell death & 3 & 0.00208138 & 0.06523 \\
\hline & GO:0009628 & Response to abiotic stimulus & 3 & 0.00217417 & 0.060715 \\
\hline & GO:0010332 & Response to gamma radiation & 2 & 0.00509224 & 0.123857 \\
\hline & GO:0031100 & Organ regeneration & 2 & 0.00575517 & 0.12707 \\
\hline & GO:0048145 & Regulation of fibroblast proliferation & 2 & 0.00774219 & 0.154437 \\
\hline & GO:0042127 & Regulation of cell proliferation & 3 & 0.0097487 & 0.177311 \\
\hline & GO:0042981 & Regulation of apoptosis & 3 & 0.01016581 & 0.172238 \\
\hline
\end{tabular}


Table I. Continued.

\begin{tabular}{|c|c|c|c|c|c|}
\hline Category & Term & Description & Count & P-value & FDR \\
\hline & GO:0043067 & Regulation of programmed cell death & 3 & 0.01036499 & 0.164649 \\
\hline & GO:0010941 & Regulation of cell death & 3 & 0.01044016 & 0.156241 \\
\hline & GO:0010212 & Response to ionizing radiation & 2 & 0.01324778 & 0.183871 \\
\hline & GO:0031099 & Regeneration & 2 & 0.0152248 & 0.198085 \\
\hline & GO:0007568 & Aging & 2 & 0.02419781 & 0.283882 \\
\hline & GO:0014070 & Response to organic cyclic substance & 2 & 0.02659589 & 0.294663 \\
\hline & GO:0001666 & Response to hypoxia & 2 & 0.02942492 & 0.308128 \\
\hline & GO:0048545 & Response to steroid hormone stimulus & 2 & 0.04197997 & 0.396429 \\
\hline & GO:0031667 & Response to nutrient levels & 2 & 0.0430572 & 0.390801 \\
\hline & GO:0010035 & Response to inorganic substance & 2 & 0.0447791 & 0.39006 \\
\hline & GO:0006916 & Anti-apoptosis & 2 & 0.04499419 & 0.379328 \\
\hline \multirow[t]{2}{*}{ Cluster 8} & GO:0008015 & Blood circulation & 2 & 0.01374926 & 0.839184 \\
\hline & GO:0003013 & Circulatory system process & 2 & 0.01374926 & 0.839184 \\
\hline \multirow[t]{3}{*}{ Cluster 9} & GO:0051384 & Response to glucocorticoid stimulus & 2 & 0.01149882 & 0.709892 \\
\hline & GO:0031960 & Response to corticosteroid stimulus & 2 & 0.01252751 & 0.490566 \\
\hline & GO:0048545 & Response to steroid hormone stimulus & 2 & 0.02818517 & 0.6393 \\
\hline
\end{tabular}

GO, gene ontology; FDR, false discovery rate.
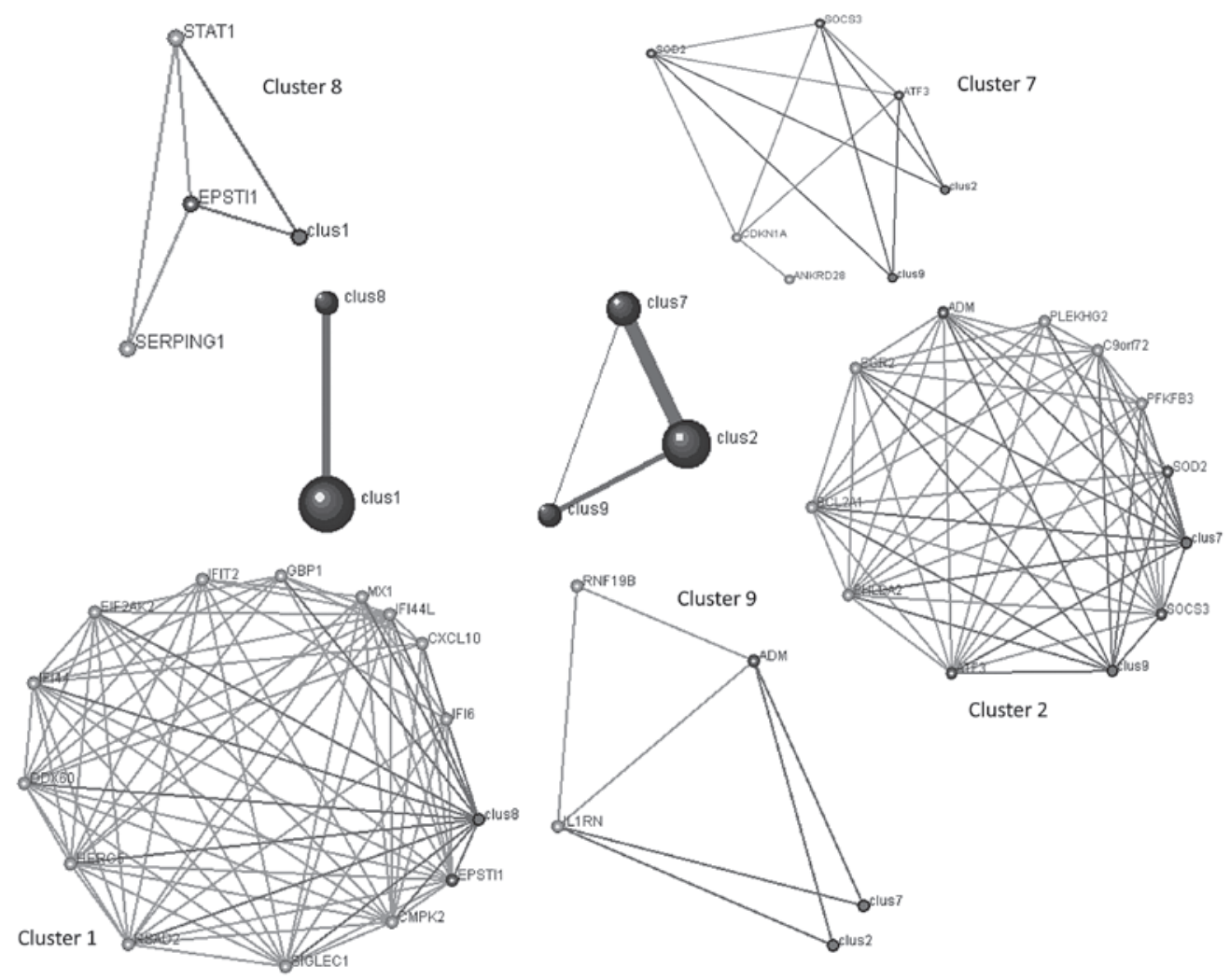

Cluster 2

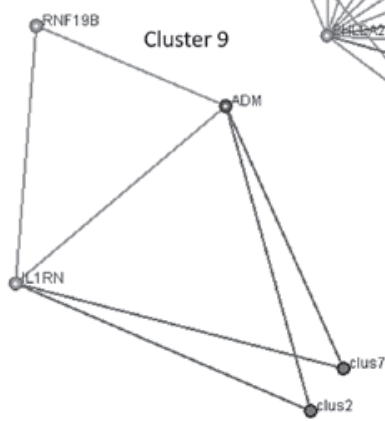

Figure 3. Graph clustering of correlated molecules in osteoporosis (threshold $r \geq 0.7$ ). Using the DPClus algorithm, 9 clusters were extracted for osteoporosis. The internal nodes of the clusters are connected by gray edges; neighboring clusters are connected by black edges.

for osteoblast differentiation, in its latent form in the cytoplasm, thereby inhibiting the nuclear localization of Runx2. This function of STAT1 does not require the Tyr 701 that is phosphorylated when STAT1 becomes a transcriptional activator (29).
The GBP1 gene is also predicted to be involved in bone metabolism or osteoclast differentiation (30) in a STAT1dependent manner (31). The sumoylation-defective STAT1 mutant exhibits increased induction of GBP1 and transporters associated with antigen presentation 1 (TAP1) transcrip- 
tion (32). The mutation in the STAT1 gene dramatically reduces the inducibility of the GBP1 and TAP1 genes by IFN (33). In this study, STAT1 and GBP1 directly interacted with each other (Figs. 2 and 3).

Chemokines have a potential role in the regulation of osteoclast functions. For example, IFN- $\gamma$-inducible protein-10 (CXCL10) is expressed in human osteoclasts with changing expression levels during osteoclast differentiation (34). CXCL10 has been suggested to contribute to osteoclastogenesis by increasing RANKL expression in $\mathrm{CD}^{+} \mathrm{T}$ cells in an animal model of rheumatoid arthritis (35). Notably, previous studies have shown that osteoblasts secrete IFN- $\beta$ in response to viral infections and that endogenous IFN- $\beta$ induces CXCL10 and IFI44L production via an IFN- $\alpha / \beta$ receptor-STAT 1 pathway $(36,37)$.

EIF2AK2 is also reported to interact with STAT1 and increase its degradation. Reduction of EIF2AK2 activity also reduces RUNX2 activity and murine osteoblast differentiation $(38,39)$. Therefore, it appears illogical that EIF2AK2 is upregulated in human osteoblasts following IFN- $\beta$ treatment which results in an inhibition of mineralization (40).

Ten genes were included in cluster 2 [ADM; early growth response 2, EGR2; BCL2-related protein A1, BCL2A1; chromosome 9 open reading frame 72, C9orf72; pleckstrin homology-like domain, family A, member 2, PHLDA2; ATF3; SOCS3; SOD2; 6-phosphofructo-2-kinase/fructose2,6-biphosphatase 3, PFKFB3; and pleckstrin homology domain containing, family $\mathrm{G}$ (with RhoGef domain) member 2, PLEKHG2], five genes were included in cluster 7 (SOCS3; SOD2; ATF3; cyclin-dependent kinase inhibitor 1A, CDKN1A; and ankyrin repeat domain 28, ANKRD28) and three genes were included in cluster 9 (ADM; interleukin 1 receptor antagonist, IL1RN; and ring finger protein 19B, RNF19B). Cluster 7 was connected with all the genes of cluster 2 and 9 by SOCS3, SOD2 and ATF3. Cluster 9 was connected with all the genes of clusters 2 and 7 by ADM and IL1RN. These findings indicate that SOCS3, SOD2, ATF3 and ADM are significant genes for responding to various stimuli, including insulin, hypoxia, nutrients, drugs, radiation and hormones regulating apoptosis.

The SOCS3 family are cytoplasmic adaptor proteins that negatively regulate various cytokine responses in leukocytes. SOCS3 overexpression augments TGF- $\beta$, TNF- $\alpha$ and RANKL-induced osteoclast formation, priming precursors to the osteoclast lineage by suppressing specific anti-osteoclastic JAK/STAT signals (41). Zhang et al demonstrated that a higher SOCS3 expression level is associated with RANKL-mediated alveolar bone loss and enhances CD11c ${ }^{+}$DC-derived osteoclastogenesis in vivo and in vitro. The reduced expression of functional SOCS3 in CD11 ${ }^{+}$DCs results in significantly lower osteoclastogenesis and dendritic cell-derived osteoclasts development during immune interactions with $\mathrm{T}$ cells, based on TRAP expression and bone resorptive activity (42). In SOCS3-deficient bone marrow-derived monocytes, the expression levels of TNF-receptor-associated factor- 6 and I $\mathrm{B}$ are drastically reduced. The receptor activation of $\mathrm{NF}-\kappa \mathrm{B}$ ligandinduced $\mathrm{I} \kappa \mathrm{B}$ phosphorylation is severely impaired, indicating that SOCS3 regulates osteoclastogenesis by blocking the inhibitory effect of inflammatory cytokines on receptor activation of the $\mathrm{NF}-\kappa \mathrm{B}$ ligand-mediated osteoclast differentiation signals (43).
ADM is a 52-amino acid peptide first described in a human phaeochromocytoma but has since been identified in numerous tissues, including the bone (44). Systemic administration of ADM stimulates the proliferation of osteoblasts and promotes bone growth (45). Treatment with ADM significantly blunts the apoptosis of serum-deprived osteoblastic cells, evaluated by caspase-3 activity, DNA fragmentation quantification and Annexin V-FITC labeling. This effect is eliminated by calcitonin-related polypeptide $\alpha$ (CGRP1) and insulin-like growth factor-I (46). The selective inhibitor of MAPK kinase (MEK), PD98059, also eliminates the protective effect of ADM on apoptosis and prevents ADM activation of ERK1/2. These data show that ADM acts as a survival factor in osteoblastic cells via a CGRP1 receptor-MEK-ERK pathway, which provides further understanding on the physiological function of ADM in osteoblasts (47).

The SOD2 gene encodes a free radical-scavenging enzyme that removes superoxidate and catalyzes the production of hydrogen peroxide. Oxidative stress is significant in the pathogenesis of osteoporosis (48). Previous studies have revealed that SOD2 is significantly upregulated in circulating monocytes at the mRNA and protein level in vivo in Chinese patients with low versus high hip BMD levels (49). Women with postmenopausal osteoporosis have significantly higher plasma SOD enzyme activity levels than those in controls (50). This indicates that SOD2 is significant in the pathogenesis of osteoporosis, promoting osteoclast differentiation, formation and activity (51).

EGR2 is a highly conserved transcription factor involved in bone remodeling. The upregulation of EGR2 is involved in the biological affinity of titanium for osteogenic cells and in the promotion of osteoblast differentiation (52). Macrophage colony-stimulating factor activates MEK/ERK and induces the MEK-dependent expression of the immediate early gene EGR2. Inhibition of either MEK1/2 or EGR2 increases osteoclast apoptosis (53). Previous studies have revealed a novel role for EGR2 in postnatal skeletal metabolism. EGR2+/- mice reveal a low bone mass phenotype. EGR2 silencing in preosteoclasts increases the expression of cFms and the response to macrophage colony-stimulating factor, leading to a cellautonomous stimulation of cell-cycle progression. Thus, the anti-mitogenic role of EGR2 in pre-osteoclasts is the predominant mechanism underlying the low bone mass phenotype of EGR2-deficient mice (54).

The osteoporotic state increases ATF3 expression in dorsal root ganglia neurons innervating L3 vertebrae (55). BCL2A1, an anti-apoptotic activated macrophage protein, is also heavily overexpressed in osteolysis patients, providing a possible mechanism for the persistence of the particle-laden cells expressing macrophage phenotype activation markers (56).

In conclusion, the present findings shed new light on the biology of osteoporosis and have implications for future research. The changes in the immune system (GBP1, STAT1, CXCL10 and EIF2AK2) and stimulus response (SOCS3, SOD2, ATF3, ADM EGR2 and BCL2A1) may be associated with osteoclast differentiation. This study provides a number of candidate genes that warrant further investigation, including DDX60, HERC5, RSAD2, SIGLEC1, CMPK2, MX1, SERPING1, EPSTI1, C9orf72, PHLDA2, PFKFB3, PLEKHG2, ANKRD28, IL1RN and RNF19B. 


\section{References}

1. Chao TH, Yu HN, Huang CC, Liu WS, Tsai YW and Wu WT: Association of interleukin-1 $\beta(-511 \mathrm{C} / \mathrm{T})$ polymorphisms with osteoporosis in postmenopausal women. Ann Saudi Med 30: 437-441, 2010

2. Lapauw BM, Taes Y, Bogaert V, Vanbillemont G, Goemaere S, Zmierczak HG, De Bacquer D and Kaufman JM: Serum estradiol is associated with volumetric BMD and modulates the impact of physical activity on bone size at the age of peak bone mass: a study in healthy male siblings. J Bone Miner Res 24: 1075-1085, 2009.

3. Andersen TL,SondergaardTE,Skorzynska KE,Dagnaes-HansenF, Plesner TL, Hauge EM, Plesner T and Delaisse JM: A physical mechanism for coupling bone resorption and formation in adult human bone. Am J Pathol 174: 239-247, 2009.

4. Marie PJ: The calcium-sensing receptor in bone cells: a potential therapeutic target in osteoporosis. Bone 46: 571-576, 2010.

5. Quinn JM, Neale S, Fujikawa Y, McGee JO and Athanasou NA Human osteoclast formation from blood monocytes, peritoneal macrophages and bone marrow cells. Calcif Tissue Int 62: 527-531, 1998

6. Kim HJ, Minashima T, McCarthy EF, Winkles JA and Kirsch T: Progressive ankylosis protein (ANK) in osteoblasts and osteoclasts controls bone formation and bone remodeling. J Bone Miner Res 25: 1771-1783, 2010.

7. Wada T, Nakashima T, Hiroshi $\mathrm{N}$ and Penninger JM: RANKL-RANK signaling in osteoclastogenesis and bone disease. Trends Mol Med 12: 17-25, 2006.

8. Kobayashi Y, Udagawa N and Takahashi N: Action of RANKL and OPG for osteoclastogenesis. Crit Rev Eukaryot Gene Expr 19: 61-72, 2009.

9. Boyce BF and Xing L: Functions of RANKL/RANK/OPG in bone modeling and remodeling. Arch Biochem Biophys 473: $139-146,2008$

10. Yao Z, Xing L, Qin C, Schwarz EM and Boyce BF: Osteoclast precursor interaction with bone matrix induces osteoclast formation directly by an interleukin-1-mediated autocrine mechanism. J Biol Chem 283: 9917-9924, 2008.

11. Goto H, Hozumi A, Osaki M, Fukushima T, Sakamoto K, Yonekura A, Tomita M, Furukawa K, Shindo H and Baba H: Primary human bone marrow adipocytes support TNF $\alpha$-induced osteoclast differentiation and function through RANKL expression. Cytokine 56: 662-668, 2011

12. Axmann R, Böhm C, Krönke G, Zwerina J, Smolen J and Schett G: Inhibition of interleukin-6 receptor directly blocks osteoclast formation in vitro and in vivo. Arthritis Rheum 60 : 2747-2756, 2009

13. McCarthy HS, Williams JH, Davie MW and Marshall MJ: Platelet-derived growth factor stimulates osteoprotegerin production in osteoblastic cells. J Cell Physiol 218: 350-354, 2009.

14. Futakuchi M, Nannuru KC, Varney ML, Sadanandam A, Nakao K, Asai K, Shirai T, Sato S and Singh RK: Transforming growth factor- $\beta$ signaling at the tumor-bone interface promotes mammary tumor growth and osteoclast activation. Cancer Sci 100: 71-81, 2009.

15. Herrera BS, Ohira T, Gao L, Omori K, Yang R, Zhu M, Muscara MN, Serhan CN, Van Dyke TE and Gyurko R: An endogenous regulator of inflammation, resolvin E1, modulates osteoclast differentiation and bone resorption. Br J Pharmacol 155: 1214-1223, 2008.

16. Nakashima K, Zhou X, Kunkel G, Zhang Z, Deng JM, Behringer RR and de Crombrugghe $\mathrm{B}$ : The novel zinc finger-containing transcription factor osterix is required for osteoblast differentiation and bone formation. Cell 108: 17-29, 2002.

17. Lei SF, Wu S, Li LM, Deng FY, Xiao SM, Jiang C, Chen Y, Jiang H, Yang F, Tan LJ, et al: An in vivo genome wide gene expression study of circulating monocytes suggested GBP1, STAT1 and CXCL10 as novel risk genes for the differentiation of peak bone mass. Bone 44: 1010-1014, 2009.

18. Chen XD, Xiao P, Lei SF, Liu YZ, Guo YF, Deng FY, Tan LJ, Zhu XZ, Chen FR, Recker RR and Deng HW: Gene expression profiling in monocytes and SNP association suggest the importance of the STAT1 gene for osteoporosis in both Chinese and Caucasians. J Bone Miner Res 25: 339-355, 2010.

19. Horan K, Jang C, Bailey-Serres J, Mittler R, Shelton C, Harper JF, Zhu JK, Cushman JC, Gollery M and Girke T: Annotating genes of known and unknown function by large-scale coexpression analysis. Plant Physiol 147: 41-57, 2008.
20. Smyth GK: Linear models and empirical bayes methods for assessing differential expression in microarray experiments. Stat Appl Genet Mol Biol 3: Article 3, 2004

21. Gentleman RC, Carey VJ, Bates DM, Bolstad B, Dettling M, Dudoit S, Ellis B, Gautier L, Ge Y, Gentry J, et al: Bioconductor: open software development for computational biology and bioinformatics. Genome Biol 5: R80, 2004.

22. Altaf-Ul-Amin M, Shinbo Y, Mihara K, Kurokawa K and Kanaya S: Development and implementation of an algorithm for detection of protein complexes in large interaction networks. BMC Bioinformatics 7: 207, 2006.

23. Fukushima A, Kusano M, Redestig H, Arita M and Saito K: Metabolomic correlation-network modules in Arabidopsis based on a graph-clustering approach. BMC Syst Biol 5: 1,2011.

24. Ashburner M, Ball CA, Blake JA, Botstein D, Butler $\mathrm{H}$, Cherry JM, Davis AP, Dolinski K, Dwight SS, Eppig JT, et al: Gene ontology: tool for the unification of biology. The Gene Ontology Consortium. Nat Genet 25: 25-29, 2000.

25. Huang da W, Sherman BT and Lempicki RA: Systematic and integrative analysis of large gene lists using DAVID bioinformatics resources. Nat Protoc 4: 44-57, 2009.

26. Clowes JA, Riggs BL and Khosla S: The role of the immune system in the pathophysiology of osteoporosis. Immunol Rev 208: 207-227, 2005.

27. Orlić I, Borovecki F, Simić P and Vukicević S: Gene expression profiling in bone tissue of osteoporotic mice. J Ind Hyg Toxicol 58: 3-11, 2007.

28. Kwak HB, Lee SW, Jin HM, Ha H, Lee SH, Takeshita S, Tanaka S, Kim HM, Kim HH and Lee ZH: Monokine induced by interferon- $\gamma$ is induced by receptor activator of nuclear factor $\kappa \mathrm{B}$ ligand and is involved in osteoclast adhesion and migration. Blood 105: 2963-2969, 2005.

29. Kim S, Koga T, Isobe M, Kern BE, Yokochi T, Chin YE, Karsenty G, Taniguchi T and Takayanagi H: Stat1 functions as a cytoplasmic attenuator of Runx 2 in the transcriptional program of osteoblast differentiation. Genes Dev 17: 1979-1991, 2003.

30. Lei SF, Wu S, Li LM, Deng FY, Xiao SM, Jiang C, Chen Y, Jiang H, Yang F and Tan LJ: An in vivo genome wide gene expression study of circulating monocytes suggested GBP1, STAT1 and CXCL10 as novel risk genes for the differentiation of peak bone mass. Bone 44: 1010-1014, 2009.

31. Cheng YS, Patterson CE and Staeheli P: Interferon-induced guanylate-binding proteins lack an N (T) KXD consensus motif and bind GMP in addition to GDP and GTP. Mol Cell Biol 11: 4717-4725, 1991.

32. Ungureanu D, Vanhatupa S, Grönholm J, Palvimo JJ and Silvennoinen O: SUMO-1 conjugation selectively modulates STAT1-mediated gene responses. Blood 106: 224-226, 2005.

33. Kovarik P, Mangold M, Ramsauer K, Heidari H, Steinborn R, Zotter A, Levy DE, Müller M and Decker T: Specificity of signaling by STAT1 depends on SH2 and C-terminal domains that regulate Ser727 phosphorylation, differentially affecting specific target gene expression. EMBO J 20: 91-100, 2001.

34. Grassi F, Piacentini A, Cristino S, Toneguzzi S, Cavallo C, Facchini A and Lisignoli G: Human osteoclasts express different CXC chemokines depending on cell culture substrate: molecular and immunocytochemical evidence of high levels of CXCL10 and CXCL12. Histochem Cell Biol 120: 391-400, 2003.

35. Lee EY, Seo M, Juhnn YS, Kim JY, Hong YJ, Lee YJ, Lee EB and Song YW: Potential role and mechanism of IFN- $\gamma$-inducible protein-10 on receptor activator of nuclear factor kappa-B ligand (RANKL) expression in rheumatoid arthritis. Arthritis Res Ther 13: R104, 2011

36. Nakamura K, Deyama Y, Yoshimura Y, Suzuki K and Morita M: Toll-like receptor 3 ligand-induced antiviral response in mouse osteoblastic cells. Int J Mol Med 19: 771-775, 2007.

37. Woeckel V, Eijken M, van de Peppel J, Chiba H, van der Eerden B and van Leeuwen J: IFN $\beta$ impairs extracellular matrix formation leading to inhibition of mineralization by effects in the early stage of human osteoblast differentiation. J Cell Physiol 227: 2668-2676, 2011.

38. Yoshida K, Okamura H, Amorim BR, Hinode D, Yoshida H and Haneji T: PKR-mediated degradation of STAT1 regulates osteoblast differentiation. Exp Cell Res 315: 2105-2114, 2009.

39. Yoshida K, Okamura H, Amorim BR, Ozaki A, Tanaka H, Morimoto H and Haneji T: Double-stranded RNA-dependent protein kinase is required for bone calcification in MC3T3-E1 cells in vitro. Exp Cell Res 311: 117-125, 2005. 
40. Woeckel VS, Koedam M, van de Peppel J, Chiba H, van der Eerden BC and van Leeuwen JP: Evidence of Vitamin D and interferon- $\beta$ cross-talk in human osteoblasts with $1 \alpha, 25$-dihydroxyvitamin D3 being dominant over interferon- $\beta$ in stimulating mineralization. J Cell Physiol 227: 3258-3266, 2011.

41. Lovibond AC, Haque SJ, Chambers TJ and Fox SW: TGF $\beta$ induced SOCS3 expression augments TNF $\alpha$-induced osteoclast formation. Biochem Biophys Res Commun 309: 762-767, 2003.

42. Zhang X, Alnaeeli M, Singh B and Teng YT: Involvement of SOCS3 in regulation of $\mathrm{CD} 11 \mathrm{c}^{+}$dendritic cell-derived osteoclastogenesis and severe alveolar bone loss. Infect Immun 77: 2000-2009, 2009

43. Yoshimura A, Sanada T, Takaki H and Ohishi M: Suppressor of cytokine signalling-1 (SOCS1) and SOCS3 regulate osteoclastogenesis by blocking inflammatory cytokine signals Nihon Riumachi Gakkai, Gakujutsu Shukai, Kokusai Riumachi Simpojiumu Puroguramu, Shorokushu 50: 15, 2006.

44. Cornish J, Callon KE, Bava U, Coy DH, Mulvey TB, Murray MA, Cooper GJ and Reid IR: Systemic administration of adrenomedullin (27-52) increases bone volume and strength in male mice. J Endocrinol 170: 251-257, 2001.

45. Cornish J, Callon KE, Coy DH, Jiang NY, Xiao L, Cooper GJ and Reid IR: Adrenomedullin is a potent stimulator of osteoblastic activity in vitro and in vivo. Am J Physiol 273: E1113-1120, 1997.

46. Cornish J, Grey A, Callon KE, Naot D, Hill BL, Lin CQ, Balchin LM and Reid IR: Shared pathways of osteoblast mitogenesis induced by amylin, adrenomedullin and IGF-1. Biochem Biophys Res Commun 318: 240-246, 2004.

47. Uzan B, Villemin A, Garel JM and Cressent M: Adrenomedullin is anti-apoptotic in osteoblasts through CGRP1 receptors and MEK-ERK pathway. J Cell Physiol 215: 122-128, 2008.

48. Abdollahi M,Larijani B, Rahimi R and Salari P: Role of oxidative stress in osteoporosis. Therapy 2: 787-796, 2005.
49. Deng FY, Lei SF, Chen XD, Tan LJ, Zhu XZ and Deng HW: An integrative study ascertained SOD2 as a susceptibility gene for osteoporosis in Chinese. J Bone Miner Res 26: 2695-2701, 2011.

50. Ozgocmen S, Kaya H, Fadillioglu E, Aydogan R and Yilmaz Z: Role of antioxidant systems, lipid peroxidation and nitric oxide in postmenopausal osteoporosis. Mol Cell Biochem 295: 45-52, 2007.

51. Deng FY, Liu YZ, Li LM, Jiang C, Wu S, Chen Y, Jiang H, Yang F, Xiong JX and Xiao P: Proteomic analysis of circulating monocytes in Chinese premenopausal females with extremely discordant bone mineral density. Proteomics 8: 4259-4272, 2008.

52. Levi G, Topilko P, Schneider-Maunoury S, Lasagna M, Mantero S, Pesce B, Ghersi G, Cancedda R and Charnay P: Role of Krox-20 in endochondral bone formationa. Ann NY Acad Sci 785: 288-291, 1996.

53. Bradley EW, Ruan MM and Oursler MJ: Novel pro-survival functions of the Kruppel-like transcription factor EGR2 in promotion of M-CSF-mediated osteoclast survival downstream of the MEK/ERK pathway. J Biol Chem 283: 8055-8064, 2008.

54. Gabet Y, Baniwal SK, Leclerc N, Shi Y, Kohn-Gabet AE, Cogan J, Dixon A, Bachar M, Guo L, Turman JE Jr and Frenkel B: Krox20/EGR2 deficiency accelerates cell growth and differentiation in the monocytic lineage and decreases bone mass. Blood 116: 3964-3971, 2010

55. Suzuki M, Ohtori S, Inoue G, Orita S, Ishikawa T, Miyagi M, Kamoda H, Eguchi Y, Arai G et al: ATF3 and GAP43 immunoreactive DRG neurons innervate the vertebral body in a rat model of osteoporosis: GP18. Spine: 2011.

56. Purdue PE: Alternative macrophage activation in periprosthetic osteolysis. Autoimmunity 41: 212-217, 2008. 